\title{
XII.
}

\section{Zu Plautus.}

Mil. Glor. arg. I. 8 :

Oberrans custos hos videt de tegulis,

Ridículis autem quasi sit alius luditur.

Das vou BC überlieferte und von Ritschl in den text aufgenommene ridiculis in v. 9 lässt sich nur durch ergänzung von modis oder dergl. erklären. Dass aber der verfasser des arguments eine so missliche ausdrucksweise angewendet haben soll, da er doch denselben sinn auf weit einfachere und deutlichere weise erhielt, wenn er schrieb: Ridicule is cett., ist sehr unwahrscheinlich.

V. 678 des genannten stuickes lautet bei Ritschl:

Líberne sunt aedes, liberum aútem esse egonet nie volo, in den handschriften: liberae sunt aedis, liber sum autem ego met. volo (cum ras. Ba, egomet volo $\mathrm{Bb}$, ego me tuuolo $\mathrm{CD}$ ) libere, zu letzterem ist in B auf dem rande 1 . bibere beigeschrieben. Nicht selten geben die marginalien des Vetus die richtige lesart, z. b. M. Gl. 668 eque stat d. i. a equest a t'que) für egestant in B, equestant in CD : Nost. 152 pila für flia in BCD, 187 slullam für stailam in $\mathrm{BCD}, 288$ aetas für aetate in BCD, Mlen. 810 tristis mit Db für tutrix in BCDa: Bacch. 344 haud mit CD für aut in B, Epid. I, 1, 35 scutariis für scrulariis, III, 3, 49 derideret für deridetur, und auch an unserer stelle liisst sich mit benutzung jenes bibere auf weit einfachere weise als von Ritschl versucht worden ist ein passender-gedanke herstellen. Fassen wir bibere, das durch einfluss des vorhergehenden liberae und liber leicht in libere verschrieben werden konnte, 
als gleich vivere, wie ja in den plautinischen haudschriften nichts gewöhnlicher ist als der wechsel von $b$ und $u$, so ist nur noch ego et für egomet zu schreiben, und gedanke wie vers ist in ordnung:

Líberae sunt aédes, liber sum áutem ego et volo vívere, frei ist mein haus, eiu freier mann bin ich und als freier mann will ich leben.

V. 996 und 997 derselben komodie sind iu folgender gestalt überliefert :

Eos nunc homines metuo mili ne obsint neve obtet (CD). opte B) uspiam.

Domosibit ac dum (BD, Domus ibil ac dum $\mathbf{C}_{\text {f huc trans. }}$ ivit atq' (CD, adq; B) huius cupiens corporist (B corporis est $\mathbf{C B})$.

Im ersten verse ist die von Ritschl nach Guyet's vorgange vor. genommene umstellung ne obsint mihi sehr wahrscheinlich und obstent für obtet unzweifelhaft richtige emendation im cod. Lip. siensis und in der ed. pr. Den zweiten vers schreibt Ritschl zum theil nach Acidalius (Domina si domo huc), zum theil nach Lindemann (Domo si illaec clam huc) und zum theil nach Camerarius (transibit quae):

Dómina si clam dómo huc transibit quae huíus cupiens corporist,

indem er iu der anmerkung zugleich noch vorschlägt: Domina si domo oder Domina domo si clam huc cett. Dass eine von diesen fassungen die ursprüngliche ist, kann man schon bei der blossen vergleichung mit der überlieferung nicht für sehr walıscheinlich halten; im besonderen fallt das müssige clam übel genug auf. Ein ganz passender sinn ergiebt sich bei höchst un. bedeutender abweichung von der überlieferung, wenn man nach der vermuthung im Lipsiensis und iu der ed. pr. schreibt: Domo si [i]bil ac dum huc transibit quae cett. Diese etwas umständliche ausdrucksweise ist dem Plautus ganz angemessen; von wem die rede ist, ist hinlänglich deutlich durch das quae huius cupiens corporist bezeichnet. Auch der vers ist in ordnung, wenn die länge der futurendung bit für Plautus wirklich durch stellen wie Men. 1160 Venibil uxor quoque eliam cett. erwiesen wird; soust bedarf es nur der einschiebung eines ea zwischen 
bal und ac, das an dieser stelle sehr leicht ausfallen konnte, uIi den vers zu vervollständigen. Freilich nimmt sich das ibit un I transibil so unmittelbar bach einander nicht sonderlich gut uls: aber letzteres ist auch gar nicht direct überliefert, sondern die hss. laben für transibil quae wie angegeben transivil alque, und diesem liegt viel näher transvitet d. i. transbilet quae als das transibit quae des Camerarius. Nach den spuren der überlieferilng scheint also der vers ursprünglich gelautet zu haben:

Domo si [i]bit ac dum huc transbitet quae huius cupiens corporist.

\section{1283 :}

(Pal.) Nauclerus hic quidemst. Py. Videlicet arcessit hac itan hic credo:

so die hss., Ritschl schreibt mit Bothe: arcessit hanc. Pal. Ila credo. Noch näher der überlieferung liegt: arcessit hanc iam hic. Pal. Credo. Das iam giebt einen sehr guten sinn: nun Prropolinices die seiner ansicht nach auch reiche Acroteleutium sicher zu haben glaubt, liegt es ihm daran, die Philocomasium, seine alte geliebte, so bald als möglich los zu werden; das erscheinen des als nauclerus verkleideten Pleusikles verheisst ihm die erfüllung dieses wunsches, noch ehe er ihn ausgesprochen liat, und so sagt er voll freude über das vermeintliche glückliche zusammentreffen der umstände: offenbar will der sie schon abholen. Die verderbniss des iam in ilam, die sich auch v. 1279 desselben stückes findet, ist auf dieselbe weise wie viele andere verlerbnisse unserer handschriften zu erklären, dass eine übergeschriebene verbesserung später von den abschreibern in den text gezogen wurde: ilam entstand ebenso aus lam wie Most. argum. 4

fileri ans fleri, 977 alio aus alo, Pseud. 1061 venitat aus ventat, $e i$

Mlerc. 599 et ei aus et, Pers. 666 irali et aus iratet. Hierauf ist wahrscheinlich auch die verderbniss von Amph. I, 1, 72 (234) Nebula conflat: cadunt vulneris vi et virium zurïckzufülıren, wo vermuthlich zu schreiben ist: Nebula conflat: cadunt vúlneris vei

curi: aus vet wurde vi el, virium schrieb dann jemand statt viri, unt wenigstens einigen sinn in die stelle zu bringen. 
Sticl. 29-33 lauten in Ritschl's ausgabe:

Pamph. Nam víri nostri domo ut ábierunt

Hic tertiust annus. Phil. Ita ut memoras;

Quom ipsi ínterea vivánt, valeant;

Ubi sint, quid agant, ecquí bene agant,

Neque participant nos neque redeunt.

Die landschriften weichen hiervon im wesentlichen nur darin ab, lass sie v. 30 bloss tertius und v. 32 ecquid agant für ecqui bene agant geben. Dass dieses ecquid agant nach dem unmittel. bar vorhergehenden quid agant unerträglich ist und nicht richtig sein kann, liegt auf der hand. Diese verderbniss scheint Ritschl's aufmerksamkeit so in anspruch genommen zu haben, dass ihm eine nicht minder schwere und zugleich nicht minder offenbare in nächster nähe entging. Die obigen verse können in der mitgetheilten gestalt doch nur folgenden sinn haben. Auf die äusserung der Pamphila, dass es schon in's dritte jahr gehe, seit ihre männer sie verlassen, erwidert Philumena, es sei so, und fügt dann als noch besonders gravirend hinzu: während sie selbst leben und gesund sind, theilen sie uns weder.mit, wo sie sind, was sie treiben, noch keliren sie zurück. Dieser ganze gedanke ist schon an sich sehr sonderbar; es kommt noch hinzu, dass er einen offenbaren widerspruch enthält : Pamphila beklagt sich, dass sie und ihre schwester schon in's dritte jahr keine nachricht über verbleiben und ergehen ihrer männer erhalten haben, und doch weiss sie, dass sie noch leben und gesund sind, ein widerspruch, der doch'schwerlich dem Plautus zuzutrauen ist. Er wird beseitigt, wenn wir mit geringer änderung der überlieferung v. 31 schreiben: Quom ISTI interea, VT VIVANT', valeant, so dass ut vivant, valeant wie ubi sint, quid agant von participant abhängen. Wie leicht VT vor Vlvant ausfallen konnte, sieht jeder; ipse für iste findet sich auch sonst noch, z. b. Aul. II, 4, 33: Quin [cum] ipsi pridem tonsor unguis demserat, Epid. 1, 1, 87: Is suo filio Fídicinam emit, quam ipse ama[vi]t, quam ábiens mandavit mihi. Ueber quom interea vergl. Men. 445: Plus triginta nátus annis [ègo] sum, quom interea loci Númquam quicquam fácinus feci peius neque scelestius, und besonders Pers. 173, eine von Ritschl arg mitgenommene stelle, die nach der überlieferung ursprünglich folgende gestalt gehabt zu haben scheint: 
Nam equidem te iam sector quintum hunc annum: quom inter[ea] iám credo,

Ouis si ín ludum iret, potuisset iam ?] fieri ut probe litteras sciret,

Quom interim tu meum ingeniúm fans atque infáns nondun etiam edídicisti,

l. l. während welcher zeit ein schaf schon hätte lesen lernen künnen, während welcher zeit du aber meinen character noch nicht vollstäudig kennen gelernt hast. Was nun v. 32 betrifft, so kann ich mich weder überzeugen, dass Ritschl mit seinem ecqui bene agant, noch dass Lomann oder Fleckeisen, jener mit ecquid ament nos, dieser mit ecqui indigean das richtige getroffen haben. Das natürlichste ist, dass Philumena ausser über ergelien, aufenthalt, thun und treiben ihres mannes und des ihrer schwester auch über den erfolg des vorhabens, um dessentwillen sie in's elend gegangen sind, nämlich sich wieder vermögen zu erwerben, nachricht wünscht, und daher halte ich es für wahrscheinlich, dass Plautus geschrieben hat:

Ubi sínt, quid agant, ecquíd pariant.

1)is für pariant überlieferte agant scheint nur irrthümliche wiederholung des unmittelbar vorhergehenden aganı zu sein.

V. 453.

Ite (A. D. ita BC) hac secundum vos me. ego hunc lacero diem,

1) Die wiederholung des iam nach einem zwischensatze scheint durch stellen wie die folgenden hinlänglich geschützt $\mathbf{z u}$ werden: Poen. V, 4, 49 , ,ita me di amabunt, út ego, si sim Juppiter, Tam hercle ego illam uxórem ducam" (Geppert mit Bothe: ,iam hercle ..illanc" cett.). Most. 15: „T u urbánus vere scúrra, deliciae popli, Rus milhi tu obiectas". Aul. III, 6, 15: "qui mihi omnis ángulos Furum impleuisti in aedibus miseró mihi"." Most. 201: "Qui pol me, ubi aetate hoc caput Colórem commutávit, Relíquit deseruitque $\mathrm{me}^{\text {" }}$ cett. Amph. 534: "Nunc tibi hanc pateram, quae ....., Alcumena tibi condono". Poen. 1320: "Sed ec cum lenonem Lycum, Bonúm virum eccum" (Geppert: ellum) video". Aul. 1, 1, 17: ,Si hercle tu ex istoc loco... excesseris aut si respexis, donicum ego te iussero, continuo hercle ego te dedam discipulam cruci“. Asin. 558: „Ed epol virtutes qui tuas nunc possit conlaudare, sicut ego possum, quae ...., Ne ille edepol" cett. Aul. IV, 10, 61: "nunc te obtestor, ut si quid ego crga te imprudens peccavi aut gnatam tuam, ut mihi ignoscas". Cas. II, 8, 75: "Ibo intro ut id quod alius condivit cocus. Ego nunc vicissim ut alio pacto condiam". Ein beispiel mit einem wie in obiger stelle wicderholten iam kann ich freilich nicht beibringen; denn die stelle Morc. 732, wo die hss. geben: „Iam si nihil usus esset iam non dicerem" ist sicherlich verdorben. 
so die hss., nur dass $A$ für vos me eqo etwas anderes zu geben scheint: Ritschl erkannte nur VOSMILGO, was er als VOSMET EGO deutet. Hat A wirklich so, worüber jetzt vielleicht Studemund genaue auskunft geben kann, so ist dies doch scliwerlich das richtige, da Plautus sonst nicht formen wie vosmet als gleichbedeutend mit den einfachen pronominalformen braucht, was hier sonst der fall wäre. Ich beschränke mich hier darauf, die plautinischen stellen mit vosmet beizubringen, um bei einer anderen gelegenheit den plautinischen gebrauch derartiger formen genauer zu erörtern: Capt. 676 : ila vosmet aiebalis. Curc. 498: Nec vobis auctor ullus est nec oosmet estis ulli. Most. 9b: atque hoc vosmet ipsi haud aliter dicetis. ib. 637: soluite vosmet igitur si saluomst. Merc. 103: Vosmet videte, quam mili valde placuerit. ib. 270: Vosmet videte ceterum quanti siem. Poen. prol, 91: Vosmet nunc facite coniecturam ceterum. Truc. II, 5, 10: vosmet iam videtis ut ornata incedo ${ }^{2}$ ). Wie Ritschl daher mit recht die freilich nur vermuthete lesart des Ambrosianus verworfen hat, so hat er auch erkannt, dass auch die lesart der übrigen hssn. nicht heil sein kann; denn eine solche stellung der präposition ist bei Plautus unerhört: er stellt in dieser weise zwischen die präpositiou und das dazu gehörige wort nur dann andere wörter, wenn die erstere nachsteht vgl. Aul. IV, 4, 27: neque tui me quicquam invenisti penes, Rud. 1348: Illud ego adoorsum si quid pecasso. Erträglich ist noch Poen. III, 4, 15: Rem advórsus populi saepe leges?, obwohl man nicht einsieht, warum Plautus nicht lieber geschrieben haben sollte: rem advorsus populi leges saepe. Ritschl schreibt nun nach der vermuthung im cod. Lips.: Ile hác secundum vos. ne ego hunc lacero diem, was gar nicht unpassend ist. Doch bietet sich eine der überlieferung noch näher liegende und dem gedanken nach noch passendere änderung dar; man schreibe:

Ite hac secundum vos. mi ego hunc lacero diem: $m i$ ist der sogenannte, dativus incommodi: s. Epid. III, 2, 8: mili cesso cum sto. Stichus hat von seinem herrn erlaubniss er. halten, diesen tag nach seinem gutdiinken zu verwenden: wemn

2) Sicherlich falsch ist wie Fleckeisen Asin. prol. 15 schreibt: "Ut vosmet alias, pariter nunc Mars adiuvet" (B. ut vos item ilias), wo "Bothe's vermuthung "Ut vos item [ut] alias" cett. nicht unwahrscheinlich ist. 
er also diesen tag lacerat d. H. loquendo perdit (cf. Asin. II, 2, 25) oder corrumpit, wie Plautus Men. IV, 2, 3 sagt, so macht e: sich mit allem rechte den vorwurf, dass er dies sich selbst zum scliaden thue. Me für mi geben die Palatini noch sonst, .. l. Bacch. 565. 684. Aul. V, 4, und in A mag wohl VOS AEI EGO stehen, wie Most. 134 die lissn. mei für mi geben.

557. Videlicet non fuisse illum nequam adulescentiam, qui ilico Ubi ille poscit denegavit dare se granum tritici.

Hercle qui aequom postulabat ille senex quandoquidem Filiae illue dederat dotem accipere pro tibicina.

Wie im cod. Lips. und in der ed. princ. für adukescentiam schon richtig adulescentem gesetzt ist, so hat auch der schreiber jenes wie der herausgeber dieser richtig gesehen, dass das non und nequam neben einander der sinn der stelle nicht verträgt. Beide latben non einfach gestrichen, und ihnen ist mit anderen Ritschl gefolgt, nur dass er mit Bothe und Loman umstellt: Videlicet nequam fuisse illum, um diesen vers mit v. 555: Videlicet parcum fuisse illum (so Guyet, die hssn. illum fuisse) senem, qui [id] dixerir. wic er demselben dem sinne nach entspricht, so auch in der ศ ortfolge möglichst übereinstimmend zu machen. Mit der til. gung des non ist allerdings der durch den zusammenhang geforderte sinn hergestellt; nur ist es rätlisellaft, wie das non in den text gekommen sein soll. Dass es ein leser oder ein absclireiber aus missverständniss hinzugefügt haben soll, ist kaum glaublich, da der sinn der stelle zu klar ist, als dass man ein missverständniss für möglich haiten könnte. Einem blossen zufalle oder muthwillen seine entstehung zuzuschreiben, ist doch much bedenklich. Sollte der felıler nicht eben so gut in nequam stecken können? Und in der that bedarf es nur einer leichten inderung zweier buchstaben dieses wortes, um alles in ordnung zal bringen; man schreibe:

Videlicet non fuísse illum aequum,

oder allenfalls auch, obwohl dies nicht unbedingt nöthig ist :

Videlicet non aequom fuisse illum cett.

Der übergang von aequum in nequam ist leicht erklärlich bei der in den palutinischen hssn. des Plautus sehr häufigen verweclıse. lung von $n, u$ und $a$. So geschrieben steht auch der vers in besserem gegensatze zu v. 560: Hercle qui aequom postulabat ille 
senex: nicht billig war augenscheinlich jener jüngling, nur bil. liges verlangte dagegen der greis. Dass in v. 558 Ritsclıl mit recht nach Acidalius denegarit für denegavit geschrieben, ergieht sich aus v. 555 videlicel parcum fuisse illum senem, qui [id] dixerit. - V. 559 und 560 schreibt Ritschl folgendermassen.

Quín hercle aequom postulabat senex quandoquidem filiae [Quam] ille dederat doten, accipere [eum voluit] pro tibicina.

Dies kann doch nur bedeuten: nur billiges verlangte der greis, da er doch wollte, dass jener (eum) die mitgift, die er (ille, sc. senex) seiner tochter gegeben hatte, für die flötenbläserin an. nehme. Wie aber dieser gedanke in den zusammenhang passen soll, ist mir völlig unbegreiflich. Der alte Antipho lässt in einem apologus einen senex von einem adulescens eine tibicina quicum cubitet verlangen, da er ihm ja seine tochter quicum cubitaret gegeben habe, und den adulescens antworten, er wolle ilm sogar gleich vier geben, falls er an einer nicht genug labe; darauf erwidert der senex, er nelıme auch vier, wenn sie sein scliwiegersolın mit dem nöthigen lebensbedarfe versehen wolle. Auf die bemerkung des Pamphilus, der senex müsse doch ein geiz. hals gewesen sein, da er noch obenein die beköstigung für die damen verlange, entgegnet Antipho, vielmelır sēi der adulescens unbillig gewesen, da er sich sofort geweigert habe, auch nur ein weizenkorn herzugeben, und sucht dinn das verlangen des senex als nur billig darzustellen. Da nuu in v. 560 von einer dos die rede ist, Pamphilus aber in seiner erwiderung v. 561 und 562 sagt, der adulescens sei vielmehr ein gescheuter mensch gewesen, der dem senex nicht eine dotata concubina habe geben wollen, so muss doch wohl Antipho die von dem senex geforderten lebensmittel für die tibicina als eine mitgift, die er billigerwweise fordern könne, bezeichnet haben. Diesen durch den eben entwickelten zusammenhang geforderten gedanken enthält die überlieferte form der in frage stelienden verse ganz klar und deutlich ausgedrückt, während diese verse in der ilınen von Ritschl gegebenen gestalt sich mit der entgegnung des Pamphilus durchaus in keinen logischen zusammenhang bringen lassen. Wie Antipho den senex sich bei seiner ersten forderung darauf berufen liess, dass er dem adulescens ja seine tochter quicum cubitaret gegeben habe, so begründet er jetzt die billig. 
icit seiner weiteren forderung durch die linweisung auf die mitwift eben jener tochter: nur billiges fürwalır, sagt er, verlangrte jener greis, nämlich, da er doch jener tochter eine mitgift gegeben hatte (quandoquidem filiae illae dederat dotem), eine mitgift auch für die libicina zu erhalten (accipere sc. dolem pro (ibicina). Zu ändern ist an der überlieferung ach nicht das ceringste: über hercle qui, vgl. Fleckeisen crit. Miscellen p. 29, iiler quandóquidem A. Speugel T. Maccius Plautus p. 77, der anch schon Ritschl's änderungen als der berechtigung entbehrend bezeichnet.

Auch v. 620 dieses stückes hat Ritschl die überlieferung olıe ausreichenden grund geändert. Dieser vers lautet bei ihm:

Tảntillum loci, ubi catellus cúbet, id satis erít mihi.

Niclit nur richtig, was wie es scheint noch niemand erkannt hat, sondern auch bei weitem drastischer ist die lesart der handscliriften :

'Tantillum loci, ubi catellus cúbet, id mihi sat, ère,' st loci.

Niclit genug, dass der parasit Gelasimus ein plätzchen, wo ein liündchen liegen mag, als für sich ausreichend erklärt, er geht in seiner demuth sogar so weit, dass er den Epignomus wie ein sclive seinen herrn mit ere titulirt. Ueberhaupt fehlt es im Sticlıus nicht an stellen, wo Ritschl's änderungen vollständig ungereclitfertigt sind. So wird v. 757 : Siquidem mihi sallaindumsl, tum vos date bibal tibicini das von Ritschl in iam geänderte tum linlänglich geschützt durch folgende stellen: Pseud. 905: si umguam quemquam di immoriales voluere esse auxilio adiulum, ıum (iam sclireibt er auch hier) me et Calidorum servatum volunt esse et lenonem exlinctum. Most. 671: Siquidem es argentum redditurus, lum bona. Poen. II, 41: si hercle isluc umquam factumst, tum me luppiter facial ut semper sacruficem nec umquam litem. il. V, 5, 1: Si ego minam non ultus /uero probe, quam lenoni dedi, tum profecto me sibi habeant scurrae ludificatui.

Pseud. 107 ist überliefert :

Spero alicunde hodie me bona opera aut haec (oder hec) meit

Tibi inventurum esse auxilium argentirium.

Ritschl schreibt zum theil nach der vermuthung im cod. Lips.: 
Spero alicunde hodie me bona opera liercle aút mala

Tibi me inventurum cett.

Abgesehen von der schwerlich plautinischeu stellung des hercle ist diese schreibart auch darum bedenklich, weil sich eine vol!. ständig genügende erklärung des mea bona opera aut mala niclıt finden lässt. Dass es, wie wohl Ritschl will, heissen kanu auf jede weise, gute oder schlechte, ein gedanke, der dem zusam. menhange ganz angemessen wäre, muss ich für sehr zweifelhaft halten; wenigstens wüsste ich diese ausdrucksweise durch kein anderes sicheres beispiel zu belegen. Ich vermuthe, dass der dichter schrieb:

Spero alicunde hodie mé (oder mea) bona opera ut [an. tid]hac

Tibi ínventurum cett.

Das selbstvertrauen, das sich in diesen worten ausdrückt, passt vortreflich zu dem character des Pseudolus ${ }^{3}$ ); auf die vergan. genheit beruft er sich ebenso v. 16: nam tu me antidhac supremum habuisti comilem consiliis luis. Die verderbniss der stelle entstand, nachdem opera aut antehac für opera ut antidhac (ante. hac für anlidhac findet sich noch sonst in unseren hssn., z. b. Ampl. II, 2, 77, wo antidhac im Vetus erst nachträglich übergeschrieben ist) geschrieben war, indem ein abschreiber das anle wegen des ähnlichen aut übersprang; in mea sehe ich eine vom rande in den text gezogene variante zu me.

3) Das die form Pseudolus keineswegs um so viel jünger als $P_{\text {setu- }}$ dulus und "rationi nullo modo conveniens" ist, wie Ritschl annimmt, hat Fleckeisen neulich nachgewiesen. Dass Plautus diese form auch wirklich gebraucht hat, dafür spricht 1) das alte, nach metrischen indicien schwerlich später als hundert jahre nach dem tode des dichters zu datirende acrostichon, in dessen fünften verse sie auch überliefert ist und dessen verfasser kaum eine andere form gebraucht haben wird, als die zu seiner zeit als die plautinische galt, 2) die wie es scheint einstimmige überlieferung der besseren handschriften ABCD im texte (Ritschl giebt zwar nur an folgenden stellen die handschriftliche lesart an , 13. 45. 326. 391. 703. 904. 1054. 1123. 1197. 1246. 1244. 1287. 1294. 1315; dass aber auch an den anderen 74. 230. 239. 348. 357. 445. 452. 465. 469. 537. 552. 697. 699. 709. 898. 1099. 1192. 1195. 1205. 1212. 1221. 1226. 1232. 1239. 1245 die hssn. nicht Pseudulus sondern Pseudolus geben, lässt sein schweigen vermuthen, da er sonst diese stellen als für seine ansicht beweisend sicherlich angeführt hätte) und auch in den scenenüberschriften, wo sie den namen überlaupt haben, bis auf fünf stellen in D (vergl. praef. ad Pseud. p. VIII). einer handschrift, die sich an alter und autorität wenigstens mit $A B$ nicht messen kann; abgesehen davon, dass diese überschriften im laufe der 
r. $205 \mathrm{sq}$. sind in BCD überliefert:

Sed nimis sum stultus nimium fui

Indoctus ne illi audeant id facere quibus ut serviaut

Suus aror cogit simul prolibet faciat

Adversum eos quod nolunt. Ca. vah tace. Ps. quid est? Male morigerus male facis mihi cum sermone huic obsonas. Ps. Taceo.

1 ist nur lesbar von id facere an: id facere - serviant bilden cinen vers, ebenso suus - nolunt oder was A für dieses wort giebt JIIINT visum est in A exslare polius quam VOLENT aut NOLINT, sagt Ritschl), und mit auslassung von vah und male morigerus die übrigen worte bis taceo, das richtig zum folgenden verse gezogen ist: der ausgang dieses letzten verses, in dem $A$ noch quo für quod wie im vorhergehenden prohibeat für prolibet geben soll, war Ritschl auch nicht mehr ganz lesbar. Das fui im ersten verse hat man bisher für die verbalform genommen, worauf man in der thut zuerst verfällt. Bei dieser aufassung kann man natürlich uicht umhin, die stelle für verderbt zu halten; Jenn dass Plautus den Calidorus, dem Ritschl mit Acidalius diese worte wie die unmittelbar vorhergehenden mit vollem rechte zugetheilt hat, in bezug auf die nämliche, von ihm eben gethane ausserung in demselben athemznge das praesens und perfect sollte haben brauchen lassen, ist nicht glaublich. Es ist aber noch eine andere auffassung von fui möglich, bei der die über-

zeit durch die willkür der abschreiber manche veränderung erfahren laben und darum ziemlich unzuverlässig sind: dass aber, wenn der dichter selbst Pseudulus schrieb, ein später entstandenes Pseudolus mit solcher consequenz im texte durchgeführt worden wäre, wäre eine gar zu merkwürdige sache; 3) haben Pseudolus Plin. n. h. XIV. 93. Fest. resp. Yerrius Flaccus an allen stellen p. $174,23.178,23.218,26$. 258, 1. 355, 28, Donat. zu Andr. I. 3. 21. I. 5. 16. II. 1. 31 ed. Lindenbr., Cledon. p. 1896 P., bei Serv. zu Aen. ed. Dan. steht I, 170. IV, 301. Pseudolus, IX, 486 Pseudulus, bei Prisc. p. 170, 14. 388, 7 cett. 1I., Diom. p. $381 \mathrm{~K}$. weist die überlieferung auf Pseudo'ss hin, Prisc. p. 139, 11. 505, 1. Diom. 339, 31 auf Pseudulus, Prisc. 205, 2. 260. 18. $4 i 1,3$. 505, 8, Diom. 379, 20 schwankt sie mehr oder weniger zwischen beiden formen. Pseudulus ist überliefert bei Cic. Cat. m. 14, $\$$. 50, in dem nicht acrostichischen argument im Ambros. v. 10 und 15, bei Gell. XX. 6 , Non. (nur p. 84,17 weist das Etdolo der hssn. auf Pseudolo lin), Charis. 77, 28. 144, 10, Probus p. 1446. 1470. P., Rufin. de metr, com. p. 384 Gaisf. und in dem alten plautinischen glossar, das aber auch wie Gell. XX. 12. Non. Prisc. Charis. Diom. Hustellarir für Mosiellaria giebt.

Philologus. XXV. Bd. 3. 
lieferung, sich wohl halten lässt; fasst man es nämlich als die dè abschen ausdrückende interjection (vgl. Pseud. 1294 phui BC, fui D, Most. 38 fue BD, ful d. i. fui C), so ist jeder anstoss besu: tigt. Die heftige ausdrucksweise des Calidorus passt sel zu der erregten stimmung, in der er sich befindet. In zweiten verse ist nempe für das unsinnige ne der lissı. eine selır walir. scheinliche vermuthung Ritschl's; dagegen vermag ich keinen zwingenden grund ausfindig zu machen, wesha!b in den folgen. den versen die worte simul prohibet (sc. amor) faciant adversum eos quod nolunt glossem sein müssen, wofür sie Ritschl erklärt. Dass sie entbehrlich sind, muss man wohl zugestehen; aber dies gieht doch noch kein recht, sie zu streichen, zumal sie sich, wie wir sehen werden, sehr wohl einer metrischen fassung fügen. Uebrigens gieht nolunt oder nolint wie malint eiven passende॥ sinn: zu jenem wäre das subject aus eos zu entnehmen, dieses hätte dasselbe subject mit faciant. Gehören die vorigen worte dem Calidorus an, so muss das oah tace dem Pseudolus, das quid est? dem Calidorus und das folgende wieder dem Pseudolus zugetheilt werden, wie auch Ritschl gethan hat. Diese ganze stelle lautet bei ihm: Ps. Táce. Cal. Quid est? Ps. Vah mile morigeru's, quór sermoni huic obsonas? Es ist also vah an eine andere stelle gesetzt, male facis mihi gestrichen und quor ser. moni für cum oder quo sermone geschrieben. Von diesen ände. rungen kann ich keine fiir hinlänglich gerechtfertigt halten; das male facis mili auch für ein glossem zu halten, das an dem ausfalle des male morigerus in A schuld war, dazu wurde Ritsclil wohl nur durch die verlegenheit, diese gauzen worte in eine passende metrische form zu bringen, veranlasst. Denn dass sich das in BCD überlieferte recht gut erklären lässt, wird woh! niemand leugnen. Weil Calidorus auf das rah race noch erst fragt quid est? und nicht sofort schweigt, macht ihm Pseudolus den vorwurf: male morigeru's, das male facis mihi cum oder quom, worauf das QVO in A hinzuweisen scheint, enthält dann die antwort auf das quid est? Der ausfall des male morigerus erklärt sich am einfaclisten damit, dass der abscbreiber irre ge. fülırt durch das zweite male das zwischen dem ersten male und facis stehende übersprang. Was nun die metrische fassung der ganzen stelle betrifft, so bedarf es weder einer auslassung noch eines zusatzes oder einer umstellung, wenn die verse in mög. 
liclıstem anschluss an die überlieferung in folgender weise abgetheilt werden:

Sed nímis sum stultus. nímiunı, fuî (oder fu),

Indoctus: $n[e m p]$ e illi audeant id facere, quibus ut serviant,

oder, worauf A hinzuweisen scheint:

Sed nimis sum stultus, nimium, fui, indoctus: nempe illi aúdeant

Id facere, quibus ut sérviant

Suus amor cogit: simul prohibet faciant advorsum eos, quod málint (oder nolunt).

Pseud. Valı tace. - Cal. Quid est? Ps. Male

Morigeru's : male fácis mili

Quom sermone huic obsonas.

V. 1048 .

Quin hic (CD, hiı B) metimur (BD, metimus $\mathbf{C}$ gradibus militariis (BCDb, mililaribus Da).

Ritschl, der im Ambrosianus von diesem verse wie es scheint, nichts hat lesen können, schreibt den anfang valde dubitanter: Quin hinc cilo imus. Dass in dem metimur oder metimus der hssı. ein verbum der bewegung stecken muss, ist offenbar, und Ja ist es mindesteus sehr walırscheinlich, dass Plautus geschrieben hat: Quin hinc meamus grádibus militáriis. Weshalb Ritschl dern nicht nur durch BC, sondern auch durch die zweite hand in D bezeugten militariis, das nur durch die erste hand in letzterer handschrift überlieferte militaribus vorgezogen hat, dafür verwag ich einen triftigen grund nicht ausfindig zu machen.

IIen. 33 lautet bei Ritschl und auch bei dem neuesten bearbeiter des stïckes :

Is púerum tollit átque in Epidamnum áuehit, die lissn. geben in der zweiten verslälfte auehitque Epidamnium. Iiornach vermuthe ich, dass der verfasser des prologs vielmehr geschrieben hat auehilque Epidamn[um] eum.

v. 892-897 sind abgesehen von unwesentlichem in folgender weise überliefert :

Sen. Quin ea te causa duco, ut id dicas mihi

Atque illum ut sanum facias. Med. Perfacile id quidemst: 
Sanum futurum mea ego id promitto fide.

Sen. Magna cum cura ego illum curari volo.

Med. Quin suspirabo plus sescenta in dies (BD. sesentia in die: $C_{j}$ :

Ita' ego illum cum cura magna curabo tibi.

Den vorletzten vers, in dem augenscheinlich ein fehler stecht, schreibt Ritschl: Quin sospilabo plus sescentos in die, und lässt ilın dann, weil er in dieser gestalt weder zu dem vorliergehenden noch zu dem folgenden verse passt, mit dem dritten den platz tauschen. Seinem beispiele ist auch Brix gefolgt. Stände die stelle so in den handschriften, so würde kaum jemand etwas daran auszusetzen liaben; dies ist aber noch immer kein beweis, dass sie auch wirklich so von hause aus gelautet hat. Vielleicht bedarf es nicht so starker lieilmittel, als Ritschl angewendet hat, um etwas dem Plautus angemessenes herzustellen. Die überlie. ferte versfolge gicbt einen ganz guten sinn, wenn der medicus im vorletzten verse dem senex, den das perfacile id quidemst lesorgt gemacht lıat, jener möchte die vermeintliche kranklıeit seines schwiegersohnes zu leicht nehmen, auf dessen magna cum cura ego illum curari volo eine probe von der phlege giebt, die er dem kranken angedeihen lassen wolle. Ein selir komisclier und zu dem prahlerischen wesen des arztes vortrefflich passender gedanke wäre es nü, wenn ihn der dichter hätte sagen lassen: Ich werde dem kranken sogar melir als sechshundertlei des tages verordnen oder eingeben, mit so grosser sorgfalt werde ich ilı dir pflegen, und einen solchen gedanken erhalten wir, wenn wir den in rede stehenden vers mit geringer änderung der überlieferung schreiben:

Quin súppeditabo plus sescenta [ei] ín die.

Suppeditare in transitiver bedeutung steht noch Asiu. 819. Dem letzten verse verhilft man zu seiner gcsetzmässigen silbenzalu] entweder durch tilgung des ego (so Ritschl) oder durch die um. stellung lia illum ego cett.

Most. 606 sq. Nescit quidem nisi faenus fabularier

Vetro te neque ego tetriorem beluam.

Vidisse me umquam quemquam quam te censeo:

so lauten diese verse im wesentlichen in BCD, und auch in A, nur dass hier Scliwarzmaun im zweiten verse für vetro te las VETOTENEQUE, Ritschl dagegen UNO . NEQUE. B giebt 
nuch anf dem rande von zweiter land $t$ velero, und darnach hat wolıl Camerarius für vetro te vermuthet velerator, was auch Rit.sclil aufgenommen hat, freilich mit der bemerkung de quo duhroare licebit. Ond in der that ist dies eine liöclist unwalırscheinliche vermuthung; denn velerator, das einen abgefeimten, Iurchtriebenen menschen bezeichnet, ist eine durchaus unpassende hezeichnung für jemand, dem man den vorwurf macht, dass er von nichts anderem als immer nur von faenus reden könne. Nirli der lesart der lissn. BCD liegt die vermuthung sehr nahe, alss in der handschrift, die ilınen zu grinde liegt, stand:

\section{Nescít quidem nisi faenus fabulárier -}

Ultro te - neque ego cett.

leber ultro te vergl. Cas. II, 8, 23: ultro te, amator, apoge te a dorso meo, Amph. I, 1, 164 und ullro isiunc Capt. III, 4, 19: ultro istum a me. In A scheint allerdings etwas wesentlich anJeres zu stehen, so dass die hssn. der anderen klasse wie läufig ciner anderen recension folgen.

V. 1134 ist in B überliefert :

Ca. Age mitte ista acto ad me ad cenam. Tr. dic ventu quid taces?

(I) geben nur isla aclo, statt der persouenbezeichnung linter cenam einen leeren raum und dicuntur umquid für dic venturum quid. Ritschl schreibt:

Call. age mitte istaec: te ád me ad cenam díc venturum. Tr. Quíd taces?

dass dem Tranio nur die worte quid laces gehören, hat er olıne zweifel richtig erkannt; dagegen scheint der anfang des verses n:och nicht ganz im reinen zu sein. Die überlieferte lesart macht es nimlich wahrscheinlich, dass Plautus schrieb:

áge mitte ista ac [c]íto ăd me ad cenam díc venturum.

Ueber die auslassung des te vgl. Mgl. 1067: quin tu huic respondes aliquid aut facturum aut non facturum. ac vor gutturalen findet sich bei Plautus, wenn auch sehr selten, vgl. Pers. 830: ac geminissumus. Bacch. 148: Ac cave malo. Asin. 779: sinul ac conspexero hodie, vor $q$ findet es sich meines wissens uirgends; Terenz dagegen scheint es nicht nur vor $q$, sondern atuch vor $c$ und $g$ gänzlich vermieden zu haben, Andr. II, 3, 8, 
wo Bentley liest: áliquam causam, quanobrem eam oppido eiciat Eiciat? 'Ac cito, ist die untadelige lesart der lissn.: quamobrem eicial oppido. Eiciat? Cito. schreiben :

Pers. 51 ist es das einfachste und wahrscheinlichste zu

Sed recipe quam primuin potes: cave fuás mi in quaestione,

die hssn. weichen nur darin ab, dass sie recipile d. i. recipe te geben. recipere steht ebenso Bacch. 234: rursum in portum re. cipimus und Merc. 498: Ergo actutum face cum praeda recipias: über quam primum potes vgl. Capt. II, 3, 88: ut quam primum possis redeas und Brix z. d. st. Ritschl schreibt: Sed récipe te quantum potest. Ebenso lässt sich auf weit einfachere weise, als von Ritschl geschehen ist, v. 256 desselben stückes herstellen. Dieser vers lautet in den hssn.:

Danunt (di meo amico hanc commoditatis copiam) argenti mutui (B. minuti CD) ut legenti opem adferam,

bei Ritschl:

Danunt, argenti mutui [compos] ut egenti opem ádferam. Es bedarf eigentlich nur des zusatzes eines buchstaben, so ist der vers in angemessener weise gefuillt:

Danunt, argenti mutui ut [e]i egenti opem ádferam.

Auch v. 300 glaube ich auf viel einfachere und wahrscheinlichere weise als Ritschl heilen zu können. In den hssn., auch in A, hat der vers im wesentlichen folgende gestalt :

Hercle íllum abisse gaúdeo. foris áperit: eccere aútern Quem convenire máxume cupiéban, egreditur íntus.

Da foris aperit nur heissen kann ,er öffnet die thür" und damit nur eine bestimmte person bezeichnet werden kann (dass Plautus etwa ianilor hätte ergänzt wissen wollen, ist nicht glaublich), während das folgende eccere aulem cett. zeigt, dass Sagaristio den offfnenden erst erkennt, als dieser aus dem hause tritt, so ist es klar, dass in dem foris aperit ein fehler steckt, was man auch schon frühzeitig erkannt hat. Selır bestechend ist die vermu. thung im cod. Lips. und in der ed. princ. foris aperif $[e]$. Darnach fordert Sagaristio an der tliür stehend und klopfend (denn mit einem aperite und dgl. hat man sich stets bei Plautus ein foris ferire oder pullare verbunden zu denken) einlass, in dem. 
seluen angenblicke öffnet sich die thür und seiu freund Toxilus tritt im gespräche mit der dienerin seiner geliebten auf die strasse. Doch hat diese vermuthung, so einfach und gefällig sie ruch scheint, ihre grossen bedenken. Einmal entspricht das 10r is aperile nicht recht dem plautinischen sprachgebrauche: wenu in den plautinischen stücken ganz allgemein um öffnung der lliï gebeten wird, so heisst es aperile Men. 674, vrgl. St. 309; uperile aliquis Merc. 130, oder aperite hoc Auph, 1020. 'Trin. 870. 1174, ecquis hoc aperil? Pseud. 1139, oder ecquis hoc aperil ostium? Ampl. 1020. Bacch. 582. Truc. III, 1, 19. Capt. 830 , ecquis istas aperit mihi fores? Most. 445, aperite hasce ambas foris Capt. 831 4 ): tritt also in solchen allgemei"I') nufforderungen zu aperire ein substantiv wie ostium oder fores, so ist dies regelmässig mit einem pronomen demonstrativum verbunden; Aul. II, 6, 1 ist eine bestimmte person angeredet, und da heisst es denn heus, Staphila, prodi atque ostium aperi whue hoc; jenes foris aperile ist also schon wegen des gegen platinischen sprachgebrauch fehlenden demonstrativen pronomen bedenklich. Dazu kommt, dass Sagaristio beim heraustreten seines frundes nach dem anfange der folgenden scene gar nicht ill der thür stand; denn dann hätte ihn dieser sogleich bemerkell müssen, während er ihn erst nach abfertigung der dienerin

4) Ich kann nicht umhin, diese von den herausgebern ziemlich gewaltsam behandelte stelle, wie sie nach der überlieferung allem anscheine nach herzustellen ist, hier herzusetzen.

V. $830(\mathrm{IV}, 2,50)$

Frg. Ileus ubi estis? ecquis hoc

Aperit ostium. Heg. Híc homo

-Ad cenam recipít se[se] ad me. Er. 'Aperite has ambás foris, Príusquam pultando ássulatim fóribus exitium ádfero.

IIeg. Perlúbet hunc hominem cónloqui: Ergásile. Er. Eirgasilum quí vocat?

Heg. Respice. Er. Fortuna quód tibi nec facit nec faciet, hoc me iubes: (vielleicht: quód tibi nec făcit nec faciet, me hoc iubes)

Sed quis est? Heg. Réspice ad me: Hegio sum. Erg. 'O mihi Quántumst hominum óptumorum óptume, in tempore \li ádvenis. Heg. Nescio quem ád portum náctus es, Ubi cenes, eó fastidis.

Dass dic letzte äusserung des Hegio an dieser stelle nicht erträglich ist, wie Brix behauptet, kann ich nicht finden. Warum soll nicht Hegio, auch nachdem sich Ergasilus zum umwenden bequemt und ihn erkannt und freudig begrüsst hat, dem parasiten noch einen vorwurf über scin vorheriges fastictire machen kônnen? 
sieht, wie er subnixis alis ambulat, vgl. v. 308. Aus diesen grüuden liat wahrscheinlich auch Ritschl jene vermuthung ver. worfen: doch was er selbst schreibt, aperilur für foris aperif ist durchaus unplautinisch: Plautus sagt stets nur aperilur ostium, Capt. 108. Men. 109. Cas. IV, 1, 21, foris Merc. 699, und dgl., nie bloss aperilur. Ich vermuthe, dass der dichter schirieb :

Hercle íllum abisse gaúdeo. foris CREPUIT: eccere autem, vgl. Aul. IV, 5, 5: allat foris crepuit: senex eccum aurum ecfert foras. Bacch. 1057 : sed crepuil foris : ecfertur praeda ex Troia. Sagaristio, der auf dem wege zu seinem freunde Toxilus ist, wird durch das knarren einer thür in seinem selbstgespräche un. terbrochen; der aus dem hause tretende ist eben der, zu den er will. Dies ist ein bei Plautus oft wiederkehrender zug.

Einer kleinen nachbesserung scheint noch v. 662 zu bedürfen. Dieser vers lautet in den handschriften:

Facium ita ut te velle (B. vellè CD) videã ut emas halue trecentis (B. trecentum CD) minis (B. minas CD).

Dass von 300 minen als kaufpreis für die virgo nicht die rede sein kann, sondern nur von 100 , lehrt der folgende vers, in dem der verkäufer auf das nimiumst des kauflustigen Dordalus seine forderung bis auf 80 minen ermässigt, und mau hat dies auch schon längst erkannt. Die lesart von CD trecentum verglichen mit der von $B$ trecentis weist deutlich darauf hin, dass urtre is

sprünglich geschrieben war: centum. Nun trecentis zu schreiben, dazu gab offenbar v. 668 die veranlassung: non edepol minis trecentis carast. Fehlerhaft ist nun noch augenscheinlich das videam, wofür Kitschl mit Acidalius video schreibt. Der bei dieser schreib. art stattfindende hiatus in der diärese des trochäischen septenar ist bei Plautus freilich olne anstoss; doch macht es das überlieferte videam wahrscheinlich, dass ihn der dichter an dieser stelle vermieden hat. Vermuthlich schrieb er:

Fáciam ita ut te vélle vide[o e]am út emas, habe centüm minis.

Merc. 131 sqq. geben die handschriften:

Char. Ecce me

Acanthio, quem quaeris. Ac. Nusquamst disciplina ignavior 
Clar. Que te res malia (B. male res D, male res C) agitant? AC. Inulta ececte (B. multae ere te CD) atque me.

Char. Quid est negotii (B. negoti: CD). Ac. Perimus.

Char. Principium inimicis dato.

Ac. At tibi sortito id optigit.

Char. Loquere id negoti quicquid est. Placide volo adquiescere.

Ritschl hat der stelle von $v 133$ an folgrende gestalt gegeben: Char. . . Ac. . . . Char. Quae te malae res [íta] agitant?

Ac. Multae bercle te[met] atque ine. Char. Quid est negoti? Ac. Periimus.

Claar. Princípium [id] inimicís dato. Ac. At tíbi sortito id optigit.

Char. Loquere id negoti quid siet. Ac. Placide: volo acquiéscere.

Ritscll's annabme einer lücke an der angegebenen stelle hat in der that viel für sicb. Acanthio hat in seinem eifer das ecce me quem quaeris seines jungen herrn überhört und fährt fort dariber zu schelten, dass ihm niemand öffnet; darauf erwartet man nach plautinischen gebrauche eine kleine erkennungsscene in der weise, dass Charinus seinen versuch, die anfmerksamkeit seines dieners auf sich zu lenken, erneuert und dieser sich dann endlich umwendet und seinen herrn erkennt. Wie die stelle überliefert ist, ist der übergang von v. $132 \mathrm{zu}$ 133 ziemlich unvermittelt und schroff. Ist wirklich etwas derartiges, wie angegeben ist, ausgefallen, so ist es doch natürlich ganz ungewiss, ob, wie Ritschl annimmt, nur ein halber vers oder ein ganzer und noch melir verloren gegangen ist. Im folgenden ist es durchaus unwahrscheinlich, dass Ritschl mit seinem multae hercle temet atque me das richtige getroffen hat, da Plautns temet ebenso wenig wie tumet gebraucht zu haben scheint. Wenigstens findet sich kein einziges sicheres beispiel dafür. Ausser dieser stelle steht diese form in Ritschl's ausgabe noch Stich. 248: Rogáre iussit temel opere maxumo und 507: Bene gesta re rediisse ambos, temet et fratrem tuum, an beiden stellen aber auch nur nach noch aus anderen gründen misslicher conjectur. Wo der begriff des tu oder te hervorgehoben werden 
soll, braucht Plautus tute und tele; ebenso ist es bei Terenz, der ausser tute noch tutimet anwendet. Doch liegt Ritschls vermuthung die richtige einsicht zu grunde, dass nach der frage des Clarinus quae te res malae agiant? in der antwort des Acantlio das te neben den me melır hervorgehoben werden muss, als es geschieht, wenn man nach $\mathrm{CD}$ schreibt multae ere te alque me, Ueberhaupt sieht diese lesart verglichen mit dem multa ececte alque me des Vetus zir sehr nach einem verbesserungsversuche aus als dass man sie für die ursprüngliche halten könnte. Nach der lesart in B liegt die vermuthung selır nalıe, dass Plautus gesclirieben hat:

Quae te res malae agitant. Multae [ae]ce te atque me. So wird das te in gehöriger weise hervorgehoben. Aecus für aequus ist in den plautiniscben lıssn. selır läufig, auf aece weist MGl. 668 das ege des B deutlich hin. Ob der vers wirklich ursprünglich ein iambischer trimeter war, ist bei der walırscheinlichkeit einer lücke natürlich unsicher: er kann auch das frag. ment eines iambischen octonars sein. Was die nach der über. lieferung folgenden drei acatalek tischen iambischen dimeter betrifft, in deren zweitem die von Lachmann vorgeschlagene einschiebung eines id nach principium den unerträglichen hiatus in selır passender weise beseitigt, so ist kein triftiger grund vorhanden, weshalb Plautus diése anordnung der metra nicht sollte zuge. lassen haben, und es ist daher gerathener, sie unangetastet zu lassen. In dem letzten der angefülırten verse muss ich Ritschl's wolıl nach Trin. 88 sed isluc negoti cupio scire quid siel (so A, die lıssn. der anderen klasse quidquid est) vorgenommene änderung loquere id negoti quid siel für eloquere id, negoti quicquid est als durchaus ungerechtfertigt bezeichnen; das überlieferte wird durch eine stelle wie Cas. III, 5, 21: sed hoc quidquid est eloquere (A ? loquere B) zur genüge geschützt.

$\mathrm{Zu}$ den stellen dieses stückes, wo Ritschl ohne ausreichenden grund die überlieferung geändert hat, gehört auch v. 291. Die lissn. geben hier: Acherunticus, senex velus, decrepilus cett. Ritschll schreibt Vetulís senex, decrepilus mit berufung auf v. 314, wo BCD nam meo quidem animo velulus descrepilus senex tantidemst, quasi sit signum piclum in pariele lıaben, A aber plane für velulus giebt. In wiefern das eigenthümliche verhältniss der beiden liandschriftenfamilien an dieser stelle, das sich bekanntlich 
vielf tch wiederholt, die von Ritschl in jener vorgenommene an. le'rung rechtfertigen soll, gestehe ich ebensowenig einzusehen, als warum er überhaupt geändert hat. Dass wenigstens an der fiir uns auffälligen verbindung senex velus die Römer keinen anstoss nahmen, zeigen folgende stellen:

Ter. Euu. 688: hic est vietus vetus veternosus senex.

Tib. I, 8, 50: in veteres esto dura, puella, seues.

Hor. Epod. 8, 3: vetus senectus.

Mart. V. 7, 3: veterem sencetam.

V. 544 ist in BCD überliefert :

Tandem ímpetravi ut egomet me corrumperem, in A las Ritschl statt impetravi - me IHPETRAVI ....... UT und schrieb darnach me egomet ut corrumperem. An der ricbtig. keit dieser schreibweise, auch wenn sie der Ambrosianus bestätigte und nicht vielmehr egomel me ut mit einem nunmehr als erlaubt nachgewiesenen hiatus in der cäsur des senars gäbe, muss ich darum zweifeln, weil Plautus wie Terenz, wenn forInen wie egomel, tule mit einem obliquen casus der entsprechenden pronominalformen ego, $t u$ cett. oder mit formen eines entspreclienden possessiven pronomen in demselbeu satze verbunden sind, egumet, tule cett. voranzustellen pflegen: vgl. Aul. IV, 9, 14: egomel me defraudavi. Amph. 457: an egomet me illic reliqui. ib. 607: quis te oerberavil? Egomet memet. M. Glor. 567: egomel me dedam libi. Merc. 930. Stich. 445: egomel me moror. Trin. 203: alque egomet me adeo cum illis una ibidem traho. Poen. V, 6, 24: quin egomel libi me addico. Pseud. 972: egomet me (novi). Stich. 581: ut egomet me hodie iugularem fame. Terent. Pliorm. 217: Mane inquam egomet me novi et peccalum meum. Trin. 663: lule pone le lalebis. Plaut. NGI. 228: lule unus si recipere ad te hoc dicis; Stich. 689: nosmet inter nos ministremus. (Ter. Hecaut. 511: ne nos[met] inter nos congruere sentiant). Pl. Amph. 416 : egomet mihi non credo. Nerc. 852: egomet mihi comes sum. 853: egomet sum mihi imperator, idem egomet mi oboedio. 854: egomet mihi fero quod usust. 'Trin. 918: egomet memini mihi. Andr. 636: proxumus sum egomet mihi. Eun. 252: imperavi egomet mihi. Ter. Heaut. 507: plus sapil quam egomel mihi. Plı. 581: te mihi fidelem esse aeque alque egonel sum mihi scibam. (Pl. Capt. 428: nec me secus umquam ei faclurum quicquam quam memet mihi,; Pl. Curc. 9: tute 
tibi puer es. Capt. 371 : tute tibi tuôpte ingenio prodes plurumum. Cist. II, 3, 21 : tule tibi indigne dotem quaeras corpore. Men. 111: tute tibi odio habeas. N. G1. 282: tute scias soli tibi. Pseud. 20: tule hinc narrato tibi. Pers. 30: si tule tibi bene esse pole pati. Truc. IV, 3, 69: lute sumpsisti tibi. Ter. Ad. 689: numquid tute prospexti tibi; PI. AuI. II, 8, 9: egomet mecum cogitare inter vias occepi. Bacch. 385: multimodis medilatus egomet mecum sum. M. Gl. 717: egomet mecum mussito. ib. 1375: egomeb mecum cogilo. Rud. 771: quom coniecturam egomet mecum facio. Ter. Andr. 82: egomet continuo mecum. Euu. 629: coepi egonet mecum inter oias cogilare. Heaut. 385: quom egomet nunc mecum in animo vitam tuam considero. Ad. 208: frustru egomet mecum hac rationes puto; Plaut. Epid. V, 1, 44: taceas tute te. cum. M. Gl. 196: Quod volutas tute tecum in corde. ib. 422 : immo edepol tute tecum (loqueris). Most. 512: quid tute tecum loquere? 551. Ter. Heaut. 200: quid tute tecum? Euu. 61: nunc tute tecum iratus cogitas Plaut. Most. 1051: ubi egomet video rem vorti in meo foro. Ter. Eun. 1024: Egomet meo indicio miser perii. Pl. Amplı. 816: Tute edictas facta tua. Baccl. 637: nunc agitas sat tute tuarum rerum. Truc. IV, 9, 13: iam livorem tule scapulis istoc concinnas tuis. Poen. I, 2, 41: ne nosmet nosira eliam vitia eloquamur. Dieselbe wortfolge bieten die band. schriften auch Pers. 573: Ferreas tule tibi impingi videas crassas cónpedis und Sticb. 293: stupplicari egomet mi (mihi die hssn.) aequom censeo, wo Ritschl geändert hat tibi tule und 'mi egomet aequom. Nicht widersprechen dem durch die angefülırten stellen erwiesenen gesetze MGl. 429: enim ne [nos] nosmel perdiderimus uspiam. Sticl. 532: Nós potius oneremus nosmet vicissatim voluptátibus (so A, Nosmet potius cett. BCD). Truc. I, 1, 59: Cum rem fidemque nosque nosmet perdimus, da nichts im wege steht, in diesen versen nos als nominativ und nosmet als accusativ zu nelımen, wohl aber

Pseud. 908: Sed ubi íllic est? sumne ego homo ínsipiens, qui lanec mécum egomet loquar solus?

Stich. 708: Tíbi propino. decumum a fonte tíbi tute inde, sí sapis.

MGL. 1117: Quin (D. qui BC) potius quŏm eam (? quai B. cum CD) túte adı̌s (tute ades CD. tu sed i B), tuam rem túte agis? (agas: BCD). 
1d. 762: Sed cesso ire intro, ne morae meis núptiis egomet siem?

Juch in allen diesen versen iasst sich äe gewöhnliche wortfolge Iurch ganz leichte umstellungen herstellen: qui egomet mecum linec loquar sólus, túte libi inde si sapis, lute luám rem agis, egomut meis nupliís siem. Die von der regel abweichende stellung recum egomel findet sich noch, wie es scheint, in v. 25 der zweiten schlussscene des Poenulus: Quid (quod BC) milh par facere sil, [me]cum (C, quom B?) egomel cónsulo. Zwar lässt sich wch hier die gewöhnliche folge mit leichter mülıe herstellen, wenn man schreilst:

Quid míli par facerest, égomet mecum consulam;

duch da diese scene aller wahrscheinlichkeit nach nicht plautinisch ist und daher was für Plautus als regel gilt nicht ohne weiteres auf dieselle ausgedehnt werden darf, so ist es wohl gerathener, das äberlieferte unangetastet zu lassen. Nach dieser atuseinandersetzung wird wohl in dem verse von dem wir ausgegangen siud, auch wenn der Ambrosianus wirklich das bieten sollte, was Ritschl vermuthet, denuoch entweder mit BCD Tandem impetravi ut egomel me corrumperem zu schreiben sein oler allenfalls: egomel me ul (' uti) corrumperem.

V. 888 schreibt Ritschl nach B :

Eut. Tuăm amicam. Char. Quíd eam? Eut. Ubi sit ègo scio. Char. 'Tune obsecro!

(D) geben am schlusse des verses tu me alumne obsecro. Allerdings ist im allgemeinen dem Vetus vor CD der vorrang einzuriiumen; alser es fehlt nicht an stellen, wo diese beiden handschriften allein dis richtige oder minder verderbtes als $B$ bieten. So sind bisweilen worte in CD erhalten, die in $B$ ausgefallen sind, und man darf daher nicht, was jene handschriften mehr als diese haben, wenn es auch noch so verderbt ist, ohne genaueste priifung und allein aus dem grunde, weil man damit nichts anzufingen weiss, verwerfen. So scheint auch an unserer stelle in dem iu me alumne von CD etwas ganz gutes zu stecken. Sehr ansprechend sind schon die vermuthungen im cod. Lips. und von Bothe: lu meam? lune obsecro? und tune aulem obsecro? Noch niilier der angegelsenen lesart liegt folgendes :

Lut. Túam amicam. Char. Quid eam? Eut. Ubi sit ego scio. Char Tune? autuma, obsecro. 
Eutychus antwortet dem aufgeregten Clarinus auf sein tune? nicht sclinell genug, deshalb fügt dieser noch hinzu autuma obsecro.

V. 997 sagt Demipho zu Lysimachus:

Quasi tu numquam qnícquam adsimile huius facti feceris, worauf dieser antwortet:

Edepol numquam: cávi nequid facerem. vix vivo miser. lch kann nicht umhin, im anfange dieses verses einen fehler zu vermuthen, da Plautus, wenn er einen satz mit numquam und edepol beginnt, sonst stets numquam edepol sagt, auch wo das metrum edepol numquam zulassen würde, vgl. Ampl. 516. 672. 1041. Asin. 279. Cas. I, 1, 40. Bacch. 515. 922. MGI. 538. 848. Most. 905. 1088. 1113 . Men. 518. Merc. 126. 438. 446. 460. Pers. 128. Pseud. 152. 507. 925. 1018. Poen. IV, 2, 65. Rud. 1288. Stich. 638. 756. Trin. 574. 1004. Truc. 11, 2, 51. Wahrscheinlich schrieb der dichter in dem Mercator. verse: Ego pol numquam. Ebenso sagt er am eingange eines satzes immer nur numquam hercle: Asin. 678. 707. Epid. V, 2, 59. 63. Men. 92. 533. MGI. 369. 581. Pers. 170. 237. 628. Rud. 470. 790. 1016. 1039. 1416. Ueberhaupt liesse sich ïber den plautinischen gebrituch von pol, edepol, hercle noch manches für die feststelluug des textes nicht unwichtiges sagen; ich will hier nur noch bemerken, dass Plautus stets namque edepol sagt, nam edepol nur, wenn andere wörter dazwischen ste. hen vgl. Capt. 604. Men. 1137. Merc. 508. MGI. 1226. Pseud. 555. Stich. 573. 663. 719. Poen. I, 2, 115, wo der neueste herausgeber unvorsiclitig gegen die hssn. nam edepol schreibt.

Truc. I, 1, 10:

Primum dum miros (B. minor C) annua $($ B. annuus $\mathbf{C})$ : is primos (B primus C) ebolust.

Ob eam tres noctis dutor (B. tutor C), cum interea loci

Aut ara aut vinum aut oleum aut triticum

Tentat benignusne an bonae frugi sies.

Für das sinnlose miros oder miror der hssn. in v. 10 hat Gronov merces vermuthet : dies giebt allerdings sinn, ist aber wegen des folgenden sehr bedenklich bei der von ihm gewollten auffassung, wenn er zur erklärung seiner vermuthung sagt: solebant enim conducere annuas meretricum operas. Denn wenn eine meretrix 
lurch eine merces annua auf ein jalır gedungen ist, so kann doch von einem anfänglichen zugestelien von tres noctes und einem $\varepsilon_{1}$ iteren hinzufügen von noctes, nachdem sich der anator als besignus potius quam frugi bonae erwiesen hat (v. 20-21), füglich nitht die rede sein. Dies muss Gronov selbst gefühlt haben, da ir am schluss seiner auseinandersetzung hinzufügt: nisi malis: primum aedium merces annua, eine sehr scharfsinnige vernuthung, die aber doch besonders wegen des zweisilbigen aedium ihre bedenken hat. Einen auch im zusammenhang der ganzen ste!le riclitigen sinn giebt das primum dum merces annua, wenn man es erklärt als eine merces, die so gross wie eine annua ist, d.h. mit ler man eine meretrix auf ein ganzes jahr miethen könnte; olu aher das blosse merces annua dies bedeuten kann, erscheint mir als sehr zweifelhaft. Eine unbedenklichere besserung des anfanges dieses verses, in dessen ausgang es natiirlich heissen muss is primus bolust, habe ich nicht ausfindig machen können; daregen kann ich wie ich glaube zur wiederherstellung der folgenden verse etwas beitragen. Für dutor oder tutor hat man utor und duco geschrieben, verinuthungen, von denen ich, so nahe sie auch liegen, keine für richtig halten kann. Diniarchus spricht hier ganz allgemein von den leiden der liebhaber, erst von r. 58 an spricht er von sich in besonderen; zu einer allgemeinen erörterung passt aber die erste person im singular schlechterdings nicht, sondern ausser passivell wendungen nur die zweite person des singular, die v. 13 und 31 angewendet ist, die dritte des singular in bezieliung auf ein vorangegangenes substantiv, wie sie im folgenden von v. 19 an in beziehung auf das vorhergehende amalor gebraucht ist, die dritte im plural und die erste pluralis, die v. $37 \mathrm{sq}$. vorkommt, wobei der redende sich als mit zu denen gehörig hezeichnet, über die er spricht. Icli gltube, das richtige ist: $O b$ eam tres noctis da[n]lur, vgl. 'Trin. 251: nox datur', über den nominativ des plural noctis $\mathrm{s}$. 1,achm. zu Lucr. p. 56. Bïcheler lat. decl. p. 15. Dass der folgende vers wie viele dieses stückes lückenhaft ist, zeigt schon dis metrum. Wie er ungefähr zu ergänzen ist, lehrt v. 19 cett., eime stelle, die sich offenbar auf diese bezieht: si id quod oratur dedit (sc. amator) atque est benignus potius quam frugi bonae, adduntur noctes. Da der amator als benignus potius quam frugi bonae von seiner dame daran erkannt wird, dass er quod oratur 
dedil, so wird das lemplare benignusne an bonae frugi siel woll vermöge des orare ut aliquid det erfolgen. Es wird dalier unsere stelle ungefälır gelautet haben:

Ob eám tres noctes dántur, quom interea loci

[Orándo oder Poscendo] aut aera aut vínum aut oleum aut tríticum

Temptát benignusne an bonae frugí sies.

Wie wenig passend die ergänzung und abtheilung der sätze in der neuesten ausgabe des stücks ist:

\section{quom interá loci}

Aut aera aut vinum aut oleum [perit] aut tríticum.

Tentat benignusne an bonae frugí sies, wird wohl auf den ersten blick einleuchten.

1, 1,27: Atque haec celimus nos damna, una (clammina CB) industria,

Cum rem fidemque nosque nosmet perdimus,

Neque parentes neu cognati seutiant.

Quos cúm celamus, si faximus cónscios (facimus conscias BC),

Qui nostrae aetati tempestive (tempestivo BC) temperint,

Ut ánteparta (unde anteparata BC) demus post partoribus

Faxím lenonum et scortorum posthác minus (plus est BC)

Et mínus damnosorum hóminum quam nunc sunt siet.

Für neque pllegrt man zu schreiben ne quid, was ja einen ganz passenden sinn giebt; elen so passend ist und noch nälıer der überlieferung liegt $N e$ quei parentes cett., vgl. Triu. 11400 : Neu qui rem ipsam posset inlellegere. Rud. 896: ne qui significem quidpiam mulierculis. Das objekt zu sentiant ergänzt sich leicht aus dem vorhergehenden. Dass in v. 70 das cum nicht ricls. tig sein kann, liegt auf der band. Das von Geppert dafuir vermuthete dum giebt nur einen richtigen sinn, wenn man es als adverb fasst; doch da Plautus dum als adverb sonst nur in verbindung mit negationen, etiam, primum und imperativen braucht, so wird diese vermuthung schwerlich das rich. tige treffen. Nimmt man aus dem folgenden celamus zu cum ein c hinzu, so liegt ganz nahe nunc, und dies bringt in den vers einen guten sinn. Im folgenden schreilt man für temperint gewöhnlich temperant, ungleich besser aber passt in den zusammenhang der conjunctiv temperent. $i$ für $e$ geben gerade in diesem stiicke die Palatini sehr oft, z. b. B. prol. 10. Alhinis prosci- 
nium, I. 1, 4 penis, II, 4, 85 ficit, II, 7, 44 loqueri, IV, 1, 5 guit, ISC I, 1, 6 exoritur, II, 1, 10 isti, II, 3, 19 nimo, II, 4, 18 li, 3.) isse, 64 pulcri, IV, 3, 22 ira.

I. 1, 75: Astaphium ancillulast (cod. est ancillula).

Cum ergo quoque etiam mili fuit commercium.

Fiir cum ergo pflegt man von alters her zu sclireiben cum ea, und dies giebt allerdings den richtigen $\operatorname{sinn}$; dass aber damit nul li der wortlaut getroffen ist, kann ich nicht glauben. Allerdings ist der Truculentus in den Palatinen in ausserordentlicl verderbter gestalt überliefert, die gewölnlichsten worte sind oft in ranz entsetzlicher weise entstellt; aber die verderbniss eines ea in ergo für möglich zu halten, fällt doch gar zu schwer, als dass man sich bei dem cum ea beruhigen könnte. Vielleicht schriel Plautus Quam erga cett. Für quam wurde quom verlesen und dies dann wie gewölnnlich in cum geändert; ergo für erga ist ein häufiger fehler, vgl. II, 7, 34 ergo eumque honorem habere. Die ausdrucksweise erga aliquem commercium est für das newöhnliche cum aliquo kann nicht mehr befremden als Aul. III, 5,12 allercatio est in aliquen.

I, 2, 68: Ast. Amator similist oppidi hostilis. Din. Quo argumentost?

Ast. Quam primum expugnari potis est amat (C, amet oder amil nach Scliwarzmann B) optumumst amicaet.

Den zweiten vers schreibt Geppert zum theil nach Bothe:

Quam prímum expugnarí potest amator optunum amicaest. Algeselien davon, dass die entstebung des hier des verses wegen unstatthaften potis est durch interpolation aus potis ungleich walırscheinlicher als aus potest ist, wie ja auch Stich. 626 statt des potis est des Ambrosianus (BCD geben potest) olne zweifel potis zu sclireiben ist, nimmt man bei dieser schreibweise noch anstoss an dem anapäst optŭmum | ămicaest. Freilich kann nicht geleugnet werden, dass Plautus solche anapästen nicht ganz gemieden hat; aber da er sie so viel als möglich vermeidet, so muss eine conjectur, die dergleichen erst in den text bringt, von vorn berein ziemlich bedenklich erscheineu, um so mebr wenn wie hier die möglichkeit vorhanden ist, gedanken wie vers auf noch andere und einfachere weise berzustellen. Melırfach nämlich wenPhilologur. XXV. Bd. 3. 
det Plautus für den begriff amator die umschreibung qui amut an, vgl. Trin. 242. Merc. IV, 4, 4. Curc. 142; setzt man diesen stellen entsprechend in dem verse des Truculentus, in dem jit die Palatinen ganz besonders häufig einzelne wörter auslassen, ror dem überlieferten amat ein qui ein, so ist alles in ordnung, nur dass natürlich noch wie schon längst geschehen für amicael ein. fach amicae zu schreiben ist.

II, 2, 22: ita me amabit sarculum

Ut egomet (A, ego me BC) ruri a me amari mavelin (A, amexarmaveum B. iau examavelim $C$ ) patulan bovem (patulum voveri BC)

Cumque ea (A. ita BC) noctem in stramentis pernoctare perpetem (BC. perpetion)

Quam tuas centum cenatas noctes mihi dono dari.

Das von Geppert im Ambrosiauus gelesene und von ilım in den text aufgenommene a me amari giebt allerdings einen sinn, und es liegt demselben auch die lesart von BC ziemlich nalıe, wenn man den in diesen handschriften nicht seltenen wechsel von $x$ und a (vgl. Truc. II, 2, 43 perext $=$ pereat, Most. 634 eaeo $=$ exeo) annimmt. Aber dennoch kann ich nicht umlin, an der richtigkeit dieser lesart zu zweifeln; denn abgesehen davon, dass man nicht einsieht, warum Plautus nicht lieber statt des ungeschickten a me amari einfach me amare gebraucht haben sollte. passt das a me amari gar nichț recht zu dem folgenden cumque ea noctem in stramentis pernoclare perpetem. Ich vermuthe nach der lesart der Palatiuen $a m[p l] \operatorname{exar}[i]$, das einen ungleich besseren sinn in die stelle bringt als das a me amari, und vielleicht auch wirklich im Ambrosianus steht. Warum statt des überlieferten tuas centum cenatas noctes (vgl. Trin. 388 gravius tuum eril unum verbum quam centum mea) Geppert tuas centies cett. zu schreiben für nothig gelialten hat, entgeht mir.

II, 6, 26: Aslaphium hat auf die frage des miles Stratophanes, ob der sohn ihrer herrin, den er für sein kind hält, ihm ähnlich sei, geantwortet, rogas? quin ubi natust machaeram et clipeum poscebal sibi, worauf jener freudig ausruft: Meus est, scio iam de argumentis. Der übrige theil des verses lautet in BC nimium quidui similest papae. Die worte nimium - similest theilt man gewölınlich der Astaphium zu, das papae dem Stratophanes, ob auf grund handschriftlicher überlieferung oder nicht, weiss ich nicht. Für quidui 
iat es lierkömmlich quidem zu schreiben; nälıer der überlieferung jieort quid [l]ui, so lass nimrum der Astaplium gehört, quid? lem miles, tui similist wieder der Astaphium. Die nöthige silben. zalbl erliält der vers, wenn meus $l$ statt meus es $l$ geschrieben wird.

II. 6, 37: Salve qui me interfecisti paéne et vita (ut ulta B. ut ultra C) et liumine.

inlerficere aliquem vila el limmine ist ein äusserst seltsamer und meines wissens sonst nicht vorkommender ausdruck. Ich vermuthe intervertisti für interfecisti, das leicht für jenes verlesen wer. den konnte; dann hat die stelle folgenden sinn: sei gegrüsst du, der Ju uich beinahe um mein leben gebracht hast.

II. 6, 62: sagt Stratophanes, als Phronesium, die sich durch keines seiner reichen geschenke zu einer freundlichen äusserung bewegen lässt, auf sein ecquid amas me? geantwortet hat: nihil ecastor neque meres:

Nilne huic sat est ?

Nemonum. (B. ne unum C) verbum quidem num dixit viginti minis.

Venire illaec posse credo dona, quae ei dono dedi.

In dem nemonum des Vetus scheint Pareus richtig ne m[i]unum erkunnt zu haben; dass zwischen ne in verbindung mit quidem und dem dazu gehörigen worte ein anderes wort tritt, ist nicht unerliört bei Plautus, wenn auch selten, vgl. MGl. 31 : ne hercle operae pretium quidemsl. onum für unum wurde in $\mathbf{B}$ auf dieselbe weise versclirieben wie v. 55 derselben scene alloli für altuli und 1, 1, 44 minos für minus, oder es steckt darin noch eine spur des alten oenum, wie dieselbe handschrift I, 2, 8 oenus für unus gieht. Für num pflegt man nunc zu schreiben, so dass also der miles sagt: nicht einmal ein einziges wort hat sie mir jetzt gesagt. Dass man sich dabei hat beruhigen können, ist unbegreiflich; denn dies kann doch der dichter den miles unmöglich haben sugen lassen, da ja Phronesium eben erst gesprochen hat. Stratoplıanes kann sich nicht darüber beklagen, dass ilım Phronesium trotz seiner freigebigkeit nicht einmal ein einziges wort gesagt habe, wohl aber, dass sie ihm nicht einmal ein einziges dankliares oder gutes wort gegönnt habe. Plautus hat dalier vermutlilich geschrieben:

Né mi unum verbùm quidem [bo]nüm díxit.

II, 7, 40 lautet in den landscliriften : 
(Geta) Quin pernam novi hominem nihil illic que est. (Pliron.) illic est.

Dass in nihil illic que est steckt nihili: illic quae[so] est? ist wohl nicht zu bezweifeln. Im anfange des verses hat man man. cherlei unwahrscheinliches vernuthet, mit am unwahrscheinlichsten ist das quin perbene novi des neuesten herausgebers. Es ist zu dem überlieferten pernam eigentlich nur ein buchstabe hinzuzı. setzen, so ist die stelle heil; man schreibe:

Geta. Quíu per[q]uam noui hominem nihili: íllic quaesost? Pliron. Illic est.

Perquam steht hei Plautus noch Baccl. 545 illorum mores per. quam medilate tenes. Dass so die worte nicht zu der versabthei. lung der neuesten ausgabe passen, darf uns wenig kümmern. denn die metrische anordnung der ganzen scene in derselben ist als gänzlich rerfehlt $\mathrm{zu}$ bezeichnen. IV, 3, 3: Rogitavi ego vos verberantis bas pendentes (IB.
laspendentis C) simul.

Dass oapulanlis ambas, wie Geppert vermuthet, das ursprïngliche ist, wird wohl niemand glauben. Sollte sich das der überliefe. rung ganz nalie liegende verberant $[e \quad h]$ is $[a m]$ bas nicht durch stellen wie Bacch. 142 praesente ibus. Eun. IV, 3, 7. Afran. (i Ribb. absente nobis. Pomp. Bonon. 47 praesenle amicis. ib. 168 praesente lestibus. Nov. 57 praesente omnibus. Acc. 428 praesente his stïtzen lassen? Mit his sind die v. 64 mit solvite angeredeten sclaven gemeint.

IV, 3, 11: Din. Etiam nunc, quid (cod. num quam) sit ne. goti falsus (cod. fassis) iucertusque sum,

Nisi quia timeo tamen ego nec quid peccavi scio.

Die worte ego nec cett. legt man gewöhnlich, wie es scheint ge. gen die handschriften, einer der beiden ancillae bei, die mit $\mathrm{Cal}$ licles aufgetreten sind, Geppert sogar beiden. Diese worte sollen dann wolil bedeuten: ich weiss nicht einmal, was ich verschuldet habe. Aber einmal ist diese stellung des nec bei Plautus unerhört, und dann reimt sich die äusserung, mag man sie nun einer oder beiden ancillae beilegen, gar nicht mit dem vorhergehenden, denn nach den worten des Callicles v. 4:

commemini quo quidque pacto sitis confessae: scio. hic nunc volo scire eodem pacto an sine malo fateamini 
hilıto sie beide schou durch schläge mürbe gemacht eiu geständn'ss abgelegt, das Callicles jetzt olıne strafmittel wiederholt halen will, und darnach kann doch keine mehr von ihnen sagen: ich weiss nicht einmal, worin ich gefehlt habe. Ebensowenig honnen diese worte dem Callicles gehören; theilt man sie dem Diniarchus zu, so geben sie in der überlieferten gestalt auch noch heinen richtigen sinn, da Diniarchus sehr wohl weiss, worin er sich vergangen hat (cf. IV, 2, 61: timeo ne malefacta antiqua mea siul ineenta omnia), wohl aber wenn man nä für nec schreibt. obwohl Diuiarchus noch nicht bestimmt weiss, ob ilın die verhandlung zwischen Callicles und den aucillae näher angeht oder nicht, kinu er sich doch nicht der furcht erwehren, da er sich seiner schuld gegen die tochter des Callicles bewusst ist.

IV, 3, 30: Call. Quíd illa cui [dono] donatast. Anc. Supposivit (cod. supposuit.) Call. Cuí? Anc. Sibi, (cod. tibi)

pro filiolo toni pro filiolo dei [del cod.] obsecro nostram

fidem.

In v. 31 theilt man gewöhnlich das erste pro fliolo noch der ancilla zu, das übrige dem Callicles, das überlieferte toni lässt uan einfach weg. Die erste liälfte dieses wortes ist allerdings woll weiter nichts als eine dittographie der letzten silbe von fliolo; wie soll aber das $n i$ in den text gekommen sein? eine frige, auf die schwerlich jemand von denen, die das ganze wort streichen, eine überzeugende antwort geben könnte. Meiner ansicht nach steckt etwas ganz gutes in dem ni. Sclıreibt man wie oben temperent für temperint so hier ne für ni und legt pro fliolune, wofür des verses wegen pro fliolon zu schreiben olne alles bedenken ist, da unsere handschriften oft genug gegen den vers ne geben, dem Callicles bei, pro filiolo der ancilla und das folgende wieder dem Callicles, so passt dies bei weitem besser zu einen verhöre, wie es hier stattfindet, als die bisherige schreibweise.

V, 5, 10 sqq. setzt Phronesium dem Stratophanes auseinunder, was zur erziehung eines kindes nöthig ist. Unter anderem ljeisst es:

Opus nutrici actute liabeat (C. B: attut abeat) veteris vini

Ut dies noctesque potet.

largiter

Fiir aclule oder allul habeat schrcibt der neueste herausgeber, um 
frühere emendationsversuche wie autem, utrem ut habeat zu über. gehen, ampullam ut habeat. Aber ampulla bedeutet bekanntlich eine kleine flasche, und wo von largiter dies noctesque potare dic rede ist, ist füglich eine kleine flasche nicht am orte; sodann ist ampulla an den stellen, wo es sich in den uns erhaltenen stücken des Plautus findet, Pers. I, 3, 44. Stich. I, 3, 77. Merc. $5,2,86$ immer nur ein geräth zur aufbewahrung von oel und ähnlichen dingen. Schliesslich hat das ampulla ut auch von seiten der überlieferung auch nicht die geringste wahrscheinlichkeit für sich. Die lesart des Decurtatus, mit der die des Vetus in wesentlichen übereinstimmt, da $c$ und $t$ in diesen handschriften unsäglich oft vertauscht sind, macht es wahrscheinlich, dass Plautus schrieb :

Opus nutrici, lácte ut habeat, veteris vini lárgiter út dies noctesque potet.

Lacle für lac sagt Plautus noch oft: vgl. MGl. II, 2, 87. Bacch. $\mathbf{V}, 2,18$. Men. $\mathbf{V}, 9,30$; über largiler mit dem genetiv vgl. Rud. 4, 4, 143.

Berlin.

o. Seyffert.

\section{Zu Cälius bei Cic. Epist. ad Famil. VIII, 1.}

§. 2: praeterea Marcellus quod adhuc nilhil reltulit de successione provinciarum Galliarum cett.: Ernesti will provinciarum streichen: aber gestrichen muss Galliarum werden, da dies für Cicero hinzuzufügen ganz überflüssig war, da der consul de provinciis zu referiren hatte, nicht bloss von Gallien, da endlich Cälius auch sonst Galliae bei der erwähnung dieser sache nicht setzt: vrgl. vor allem ep. 4, 4, dann auch 5, 3. 9, 2. 11, 3. 13, 2: anders verhält es sich mit 5, 2. 8, 4. 9. Aus Baiter sehe ich (praef. p. xxxi1) so eben, dass auch Pluygers Galliarum auswerfen will.

In $\$$. 4 heisst es, ego . . . non sum commolus et hoc men dacio, si qua pericula libi impenderent ut defungeremur oplati: da hat et gar keinen sinn: es ist zu streichen, dafür aber vor si qua ein sed einzuschieben, da zwischen si qua cett. und dem vorhergehenden gar keine verbindung nach der handschriftlichen von allen herausgebern beibchaltenen $\mathbf{L A}$ stattfindet.

Ernst oon Leutsch. 